\title{
Universal Landau Pole
}

\author{
A. A. Andrianov, ${ }^{1,2, *}$ D. Espriu, ${ }^{2, \dagger}$ M. A. Kurkov, ${ }^{3,4, \ddagger}$ and F. $\operatorname{Lizzi}^{2,3,4, \S}$ \\ ${ }^{1}$ Department of Theoretical Physics, Saint-Petersburg State University, St. Petersburg 198504, Russia \\ ${ }^{2}$ Departament Estructura i Constituents de la Matèria, and Institut de Ciències del Cosmos (ICCUB), \\ Universitat de Barcelona, 08028 Barcelona, Catalonia, Spain \\ ${ }^{3}$ Dipartimento di Fisica, Università di Napoli Federico II, Napoli, Italia \\ ${ }^{4}$ INFN, Sezione di Napoli, I-80126 Napoli, Italia
}

(Received 27 February 2013; published 1 July 2013)

\begin{abstract}
Our understanding of quantum gravity suggests that at the Planck scale the usual geometry loses its meaning. If so, the quest for grand unification in a large non-Abelian group naturally endowed with the property of asymptotic freedom may also lose its motivation. Instead, we propose a unification of all fundamental interactions at the Planck scale in the form of a universal Landau pole, at which all gauge couplings diverge. The Higgs quartic coupling also diverges while the Yukawa couplings vanish. The unification is achieved with the addition of fermions with vector gauge couplings coming in multiplets and with hypercharges identical to those of the standard model. The presence of these particles also prevents the Higgs quartic coupling from becoming negative, thus avoiding the instability (or metastability) of the standard model vacuum.
\end{abstract}

DOI: 10.1103/PhysRevLett.111.011601

PACS numbers: $12.10 . \mathrm{Kt}, 11.10 . \mathrm{Hi}$

Under the renormalization group flow, the coupling constants of the three fundamental gauge interactions behave quite differently [1]. While the couplings of the non-Abelian interactions, weak and strong, constantly diminish as the energy increases, the coupling of the Abelian interaction grows, and eventually diverges, a phenomenon usually referred to as a Landau pole [2].

This results from a direct extrapolation of physics at present energies. The existence of new particles, or in general new physics, alter this behavior. For some time, it was thought that the three interactions coincided at a single scale, and this was interpreted to signal the presence of a nonAbelian grand unified group. Present accurate data show that this triple coincidence does not happen in the absence of new physics. The weak and hypercharge couplings are equal at a scale of the order of $10^{12} \mathrm{GeV}$, hypercharge and strong at around $10^{14} \mathrm{GeV}$, and the two non-Abelian couplings meet around $10^{17} \mathrm{GeV}$.

Continuing the flow of the three couplings beyond the previous scales would give rise to a "weak" force actually stronger than the "strong" one, with the Abelian coupling overtaking both of them. If one continues the running of the hypercharge coupling, it diverges at the finite, albeit extremely large, scale of $10^{53} \mathrm{GeV}$. Even though the calculations are done perturbatively at a finite loop order and the value of the scale where the pole occurs is therefore not to be trusted numerically (since the perturbative expansion will have broken before), the qualitative behavior will however remain: for a nonasymptotic free theory at some energy, there will be a pole.

Also relevant is the behavior under the renormalization flow of the quartic Higgs coupling $\lambda$ and of the Yukawa couplings of the top $y_{t}$, which is the largest of the fermion couplings and therefore dominates at high energy. They both decrease, but while $y_{t}$ remains positive, $\lambda$ becomes negative in the presence of a relatively light Higgs boson mass around $\sim 125 \mathrm{GeV}$ [3]. This signals an instability, or at least a metastable phase, of the theory.

The idea of the unification of the forces is very appealing. A grand unified group guarantees the presence of asymptotic freedom and consequently the ability to describe particles and fields at arbitrarily small distances. However, we know that in nature there is also gravity and before the Planck scale, around $m_{p}=10^{19} \mathrm{GeV}$, the onset of quantum gravity will certainly alter the picture in a substantial way. Models of emergent gravity (see, e.g., [4]) indicate that there may be a "smallest distance" below which the very notion of length may not exist. In any case, dramatic quantum gravity effects-perhaps a string theory-are likely to manifest themselves at around the Planck scale and it is not obvious at all why one should expect quantum field theory to remain perturbatively valid at or beyond the Planck scale. Then, the philosophical necessity for asymptotic freedom at the most fundamental scale weakens considerably.

In this Letter, we want to put forward another type of unification. Namely, the proposal that all coupling constants, as well as the Yukawa couplings and the quartic Higgs coupling have a singularity at an energy of the order of the Planck mass. This common singularity, which we term universal Landau pole (ULP) may be interpreted as signaling the onset of a phase transition to radically new physics. The nature of the "high energy" trans-Planckian phase is not known, there would probably be some sort of quantum space-time and hypotheses abound. The existence of a common singularity might hint that this new phase 
could be weakly coupled, but in a completely different set of variables. We assume that singularity at the transition shows up as a pole in all gauge couplings and the quartic coupling, and a zero for the Yukawa coupling. In the following, we will see that the model presented here also solves the potentially disastrous instability of the Higgs potential [5].

The recent Large Hadron Collider measurement [3] of the Higgs boson mass around $125 \pm 1 \mathrm{GeV}$ together with the absence of new physics indicate that the quartic coupling of the Higgs self-interaction may become negative at an energy as low as $10^{8} \mathrm{GeV} \mathrm{[6]} \mathrm{suggesting} \mathrm{an} \mathrm{instability}$ of the theory. In terms of the effective potential, this is tantamount to a negative quadric term; therefore, the potential is not bounded from below.

In the scenario we propose, the presence of new particles solves the stability problem, and at the same time generates the ULP. In order to achieve this, the new physics must hasten the running of the Abelian coupling towards the pole, lowering it from $\sim 10^{53}$ to about $10^{19} \mathrm{GeV}$, modify the running of the quartic coupling, and avoid the appearance of problems in known physics. In the following, we show a model where we achieve the objectives listed above. The aim of this exercise is to show a reasonable possibility where a ULP with the required properties manifests itself. While we do not claim that our solution is unique, we want to stress at the beginning that finding a scenario which is not in contradiction with known physics, and at the same time fulfills some requirements (specified below) is not easy. Furthermore, if we require that the new scenario solves the instability of the Higgs potential then the possibilities are reduced to basically one scenario.

The possibility that a Landau pole may be present at the Planck mass is not totally new. The authors of [7] used this hypothesis to set bounds for the number and masses of quarks and leptons. Later, the possibility of a unification at strong coupling has been studied in the context of the grand unified theory and supersymmetry (SUSY), see, e.g., [8,9], and references therein for a review. We follow a different line: ULP is related with physics at the Planck scale and we do not introduce a new gauge group nor SUSY, and no new gauge or Higgs fields. Another interesting proposal, which has some similarity with ours, relies on the possible existence of a non-Gaussian UV fixed point. This conjecture arises from the possibility of gravity being an asymptotically safe theory [10] and assuming that all other interactions unify at the same nonperturbative fixed point (see recent discussion in [11]) and, remarkably enough, this conjecture may have interesting implications on the quartic coupling as well. This latter proposal does not assume a radical new theory for gravity-just an hypothetical nonperturbative completion of the usual theory.

From now on, we will adhere to the minimal hypothesis of the existence of a ULP. In this Letter, we will present our calculations at the one loop level. Present theoretical knowledge would, in principle, enable us to perform the calculation up to three loops [12] and we will present a full analysis of the two-loops calculation in a forthcoming work [13]. The two-loop result will not significantly be different from the one presented here because we perform the analysis in the region below the first new threshold (where the top Yukawa coupling is largest $\sim 1$ and perturbation theory in the scalar sector worst) with the full two loops theory, and used the one-loop approximation only above this first threshold where $Y_{t}$ is smaller.

At one loop, the running of the couplings is given by simple equations

$\frac{d g_{i}(t)}{d t}=\beta_{i}(t), \quad \beta_{i} \equiv \frac{1}{16 \pi^{2}} g_{i}^{3} b_{i}, \quad t \equiv \log \frac{\mu}{\mathrm{GeV}}$,

where $i=1,2,3$ represents the $\mathrm{U}(1), \mathrm{SU}(2)$, and $\mathrm{SU}(3)$ couplings, respectively. The presence of new particles will alter this running. At one loop, the behavior of

$$
\frac{1}{\alpha_{i}} \equiv \frac{4 \pi}{g_{i}^{2}}
$$

are linear. The presence of new particles just alters the slope of straight lines.

We now present our model, explicitly spelling out the constraints we impose. We require the model to have the standard model (SM) gauge group and particles coming in generations with the same quantum numbers as the usual ones. This ensures, for example, that there will be color singlets made of three fermions. We will assume that the various particles have masses such that they contribute only when a particular threshold of energy is reached. The full evolution is, therefore, given by a set of straight segments and the solution is found matching the boundary conditions. To differentiate the new from the old particles, we will call them "quarkons" and "leptos." To avoid problems with anomalies, and the introduction of new Higgs-like particles, all new particles are "vectorlike" Dirac particles, but they maintain the representations of the known gauge groups. This also avoids that the presence of new particles coupled to the Higgs boson could actually render the instability of the quartic term more pronounced. In fact, recent LHC results [14] severely constrain the possibility of having a (standard-model-like) fourth generation coupled to the Higgs particle, no matter how large its mass. However, new heavy fermions appearing in vector representations are still largely unconstrained.

We emphasize that all quarkons and leptos are vectorlike particles and that they do not get their masses through the Higgs mechanism. In particular, one can consider them to have Dirac masses. Thus, the Higgs boson Lagrangian has the form of the SM and therefore, the one-loop RG flow of quartic coupling and of Yukawa couplings are fully controlled by the behavior of gauge couplings. Baryon and lepton charges for new fermions are conserved separately. Quarkons are SU(3) triplets and leptos are SU(3) singlets. 
They both come in two kinds: $\mathrm{SU}(2)$ doublets, which we will call $L$ quarkons and $L$ leptos, with the hypercharges of left quarks and left leptons, respectively; and SU(2) singlets, $R$ quarkons, with the hypercharge of right-handed quarks, and $R$ leptos carrying the hypercharge of right electrons. However, $R$ leptos, which are singlets for all SM groups (like right neutrinos), could be present in principle, but, since they do not contribute to the running of the gauge couplings, we can ignore them.

For the $U(1)$ gauge coupling $g_{1}$, the constant $b_{1}$ is given by:

$$
\begin{aligned}
b_{1}= & \frac{41}{6}+\frac{2}{3} N_{L \text { leptos }}+\frac{4}{3} N_{R \text { leptos }} \\
& +\frac{2}{9} N_{L \text { quarkon }}+\frac{20}{9} N_{R \text { quarkon }} .
\end{aligned}
$$

For the $S U(2)$ gauge coupling we have:

$$
b_{2}=-\frac{19}{6}+\frac{2}{3} N_{L \text { leptos }}+2 N_{L \text { quarkon }}
$$

For the $S U(3)$ gauge coupling

$$
b_{3}=-7+\frac{4}{3}\left(N_{L \text { quarkon }}+N_{R \text { quarkon }}\right) .
$$

The integers $N$ in these formulas refer to the number of quarkon and leptos multiplets contributing to beta functions.

Since the coefficients are piecewise constant, and change at the energies representing the scale at which the new particles appear, it is possible to do a systematic search. We have imposed as a boundary condition of the differential equation that $1 / \alpha_{i}=0$ at the Planck scale $m_{p}$. In any case, the model cannot be trusted at energies approach $m_{p}$ for more than one reason. The perturbative approach will have broken down, not to speak of the one loop approximation, and, moreover, gravitational effects could not be ignored. Our setting a precise boundary condition, giving a common pole at a particular scale, is, therefore, just expedient for describing a common pole that the present theoretical tools cannot properly describe.

The other low energy boundary conditions are given by the experimental values: $\alpha_{s}=0.1184, g_{1}=0.358729$, $g_{2}=0.648382, g_{3}=1.16471, y=0.937982$, and $\lambda=$ 0.125769 for $M_{H}=124 \mathrm{GeV}$ at the scale of the top mass $\mu=M_{t}=172.9 \mathrm{GeV}$. These values are insensitive to $M_{H}$ in the range 124-126 GeV. Since the equations are linear (at one loop), the results presented here are quite "robust" for slight changes in the boundary condition, both in the low energy and pole regions.

In principle, there would be four kinds of particles that switch on at four scales, and the boundary conditions at the intermediate scales impose three constraints. We require the scales to be between the TeV region and the ULP, and that the evolution is monotonic (the curves must not intersect themselves). This results in the only allowed order of the different thresholds as one goes up being the following: $L$ quarkons, $R$ quarkons, $L$ leptos, $R$ leptos. If one requires the switching on of the leptos to be at the same scale, one finds solutions. On the contrary, setting the quarkons at the same scale does not provide a solution. This enables us to reduce the number of parameters to three, with three equations, therefore finding a unique solution. Since the scale for the leptos must be larger than the one of quarkons, and therefore closer to the Planck scale, the possibility of splitting the two scales of the leptos gives just a little uncertainty at very high energies. We were also able to fix the number of generations. For three generations or less, there is no solution in the physical range. For more than four generations, the quartic coupling develops an instability.

Therefore, we consider that there are four identical "generations." With the given constraints the particles must be at the following scales:

(i) At $5.0 \times 10^{3} \mathrm{GeV}$ the $L$ quarkons $\left(N_{L \text { quarkon }}=4\right)$.

(ii) At $3.7 \times 10^{7} \mathrm{GeV}$ the $R$ quarkons $\left(N_{R}\right.$ quarkon $\left.=4\right)$.

(iii) At $2.6 \times 10^{14} \mathrm{GeV}$ the $L$ and $R$ leptos $\left(N_{L \text { leptos }}=N_{R \text { leptos }}=4\right)$.

It is interesting to remark that the lowest threshold in this minimal ULP model is tantalizingly close to the reach of the LHC but certainly not excluded by present data.

In Fig. 1, we show the running of the gauge coupling. (the initial running shown is actually made with the two-loop equation). One can see that the hierarchy of the couplings is respected; the strong force remains stronger

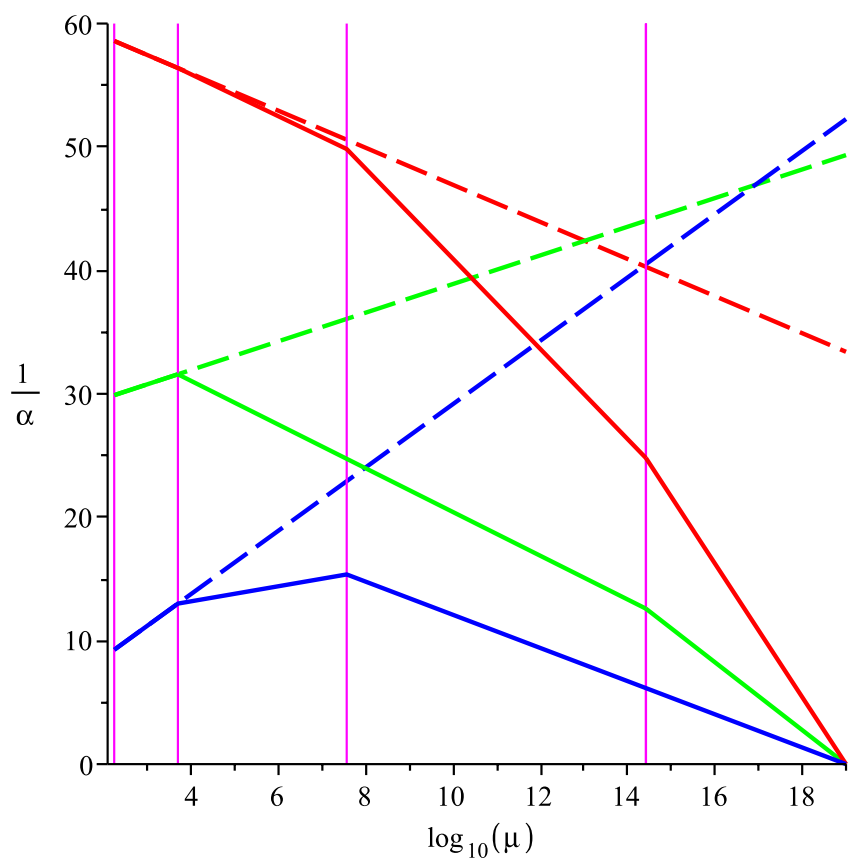

FIG. 1 (color online). The running of $\alpha_{i}$, the inverse of the gauge couplings. The dotted lines are the runnings in the absence of quarkons and leptos. The $\alpha_{i}$ are in descending order as $i$ increases. 
than the weak. The scale at which there is the appearance of the new particles is larger than the experimental bounds on the presence of new fermions, but not too much. This scenario shows that the ULP may exist with new physics at energies within reach. Other solutions are possible, and we will comment on them in [13], although the qualitative features of these alternative options are similar to the one presented here.

The running of the gauge couplings affects the other couplings we considered. As far the top Yukawa coupling is concerned, the equation is

$$
\beta_{y}^{(1)}=\frac{1}{(4 \pi)^{2}} y\left(-\frac{9}{4} g_{2}^{2}-\frac{17}{12} g_{1}^{2}-8 g_{3}^{2}+\frac{9}{2} y^{2}\right) .
$$

Solving the differential equation, we obtain Fig. 2. The coupling is undistinguishable from the standard model for energies up to $10^{6} \mathrm{GeV}$, and vanishes at the ULP.

The quartic coupling equation is

$$
\begin{aligned}
\beta_{\lambda}^{(1)}= & \frac{1}{16 \pi^{2}}\left(24 \lambda^{2}-6 y^{4}+\frac{3}{4} g_{2}^{4}+\frac{3}{8}\left(g_{2}^{2}+g_{1}^{2}\right)^{2}\right. \\
& \left.+\left(-9 g_{2}^{2}-3 g_{1}^{2}+12 y^{2}\right) \lambda\right) .
\end{aligned}
$$

Its solution, given the couplings we calculated earlier, is shown in Fig. 3. We see that the quartic coupling for our choice of new particles comes close to vanishing, but never actually becomes negative. At the ULP, the coupling diverges. Therefore, the potential term will dominate over the other terms. The mass parameter remains finite with a regular behavior while the evolution is in the perturbative regime.

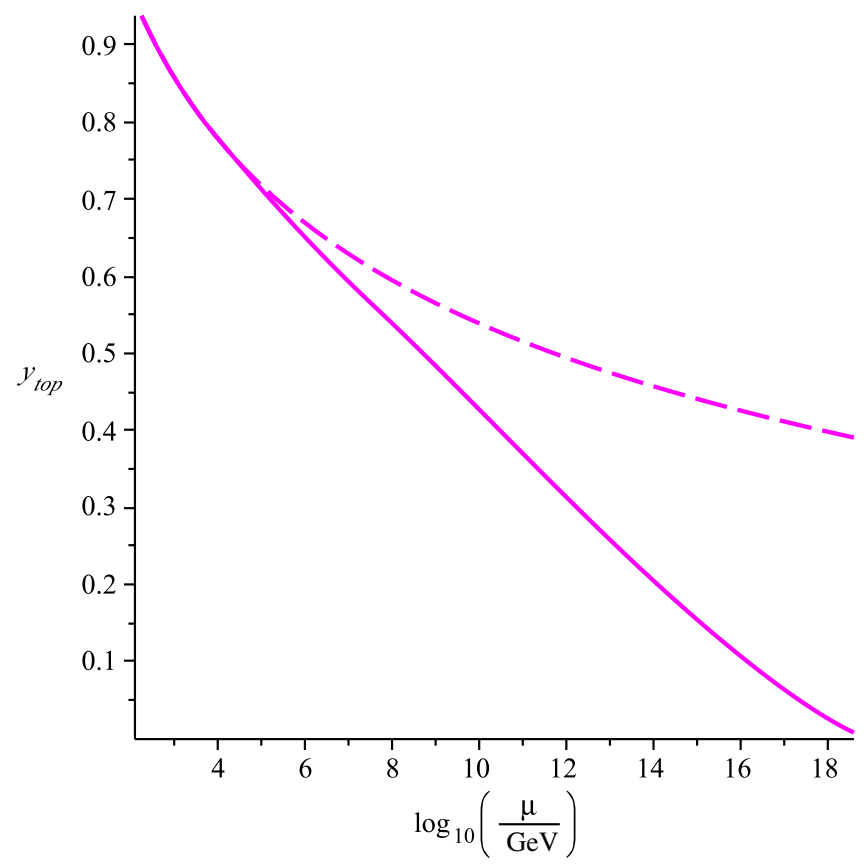

FIG. 2 (color online). The running of $y_{t}$. The dotted line is the SM case.
We expect that taking into account two-loop corrections does not affect the position of the successive thresholds dramatically. Indeed, from the SM, we know that the gauge running remains almost unchanged after taking into account two and even three loops: the renormalization group trajectories almost coincide with the lines of constant slope obtained in a one loop computation [12]. Since all our scales lie in a perturbative regime, one expects to have the same property in the ULP scenario. Thus, we conclude that one can trust the one loop determination of the masses of quarkons and leptos.

The scenario described in this Letter may give hints to aspects of the trans-Planckian phase where the kinetic term becomes negligible and the propagator "freezes," and the Higgs boson (which might be composite) decouples. This suggests that gauge bosons may possibly be effective (probably composite too) degrees of freedom. Gravity will play a dominant role, but the absence of propagating degrees of freedom suggests a "geometry" without points, with spacetime possibly described by a noncommutative geometry [15] and/or replaced by a pregeometric entity, such as the spin foam and spin networks of quantum gravity [16]. In proposals such as [4], even gravity is described entirely in terms of fermions, which may describe the whole physical world if a scenario like a ULP is realized.

It could well be the case that the onset of gravity corrections renders the ULP we advocate in this Letter nonsingular. Indeed, gravity being nonrenormalizable will require higher-dimensional operators with more

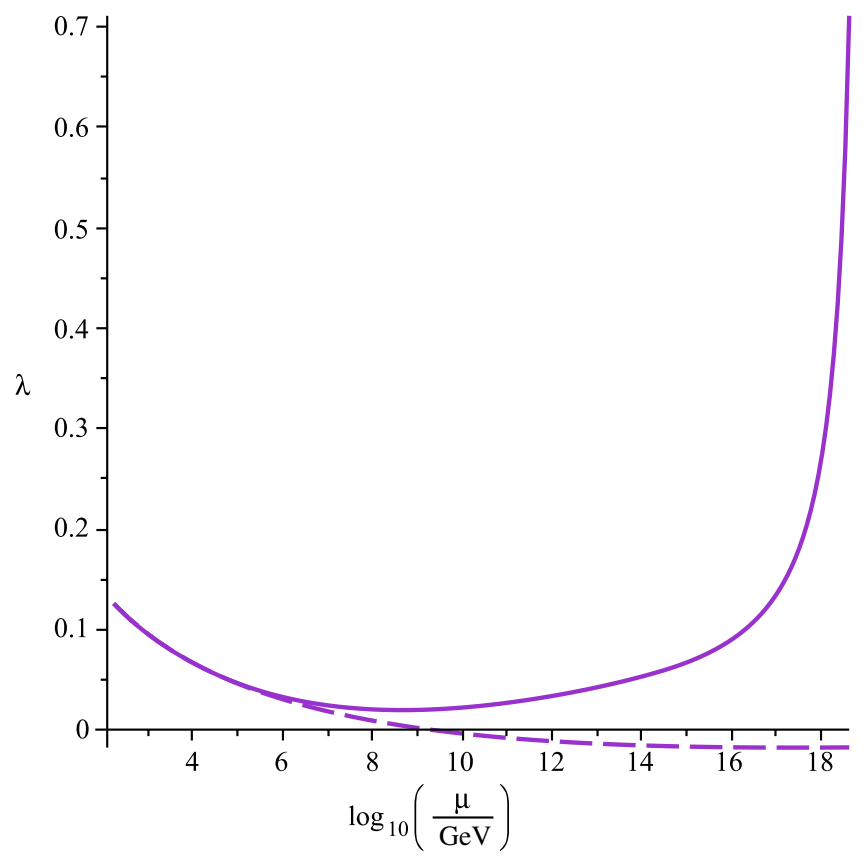

FIG. 3 (color online). The running of quartic coupling of the Higgs field. The dotted line shows the instability that the standard model develops in the presence of a Higgs mass of 124-126 GeV. 
derivatives to render the theory finite. In particular, we expect dimension six kinetic terms like

$$
\frac{\gamma}{2 M_{P}^{2}} \operatorname{tr}\left(D_{\mu} W^{\mu \nu} D_{\mu} W_{\nu}^{\mu}\right)+\cdots
$$

This would correspond to a renormalization of the gauge coupling induced by gravity of the form

$$
\frac{1}{g^{2}\left(p^{2}\right)} \simeq \beta_{0} \log \frac{m_{P}^{2}}{p^{2}}+\gamma \frac{p^{2}}{m_{P}^{2}} .
$$

As shown in [11], gravitational corrections may drive the ULP towards a new fixed point.

The renormalization flow of the various constants, especially in view of the new data coming from the LHC, may be an essential tool for the understanding of physics at the gravitational frontier.

We thank M. Zoller for a private communication regarding the choice of initial conditions. A. A., D. E. and F. L. are partially supported by projects FPA2010-20807, 2009SGR502 and CPAN (Consolider CSD2007-00042). This work has been made possible by the INFN-MCINN bilateral agreement AIC-D-2011-0815. A. A. and M. A. K. are also supported by Grant No. RFBR 13-02-00127 and by SPbSU Grant No. 11.38.660.2013.

*andrianov@icc.ub.edu

†espriu@icc.ub.edu

†kurkov@na.infn.it

§fedele.lizzi@na.infn.it

[1] J. Beringer et al. (Particle Data Group), Phys. Rev. D 86, 010001 (2012).

[2] L.D. Landau, A. A. Abrikosov, and I. M. Khalatnikov, Dokl. Akad. Nauk SSSR 95, 497 (1954); 95, 773 (1954); 95, 1177 (1954); L.D. Landau, I. Ya. Pomeranchuk, Dokl. Akad. Nauk SSSR 102, 489 (1955).
[3] G. Aad et al. (The ATLAS collaboration), Phys. Lett. B 716, 1 (2012); S. Chatrchyan et al. (The CMS collaboration), Phys. Lett. B 716, 30 (2012).

[4] J. Alfaro, D. Espriu, and D. Puigdomenech, Phys. Rev. D 86, 025015 (2012).

[5] N. V. Krasnikov, Yad. Fiz. 28, 549 (1978); P. Q. Hung, Phys. Rev. Lett. 42, 873 (1979); H. D. Politzer and S. Wolfram, Phys. Lett. 82B, 242 (1979); 83B, 421(E) (1979).

[6] J. Elias-Miro, J. R. Espinosa, G. F. Giudice, G. Isidori, A. Riotto, and A. Strumia, Phys. Lett. B 709, 222 (2012); F. Bezrukov, M. Yu. Kalmykov, B. A. Kniehl, and M. Shaposhnikov, J. High Energy Phys. 10 (2012) 140.

[7] L. Maiani, G. Parisi, and R. Petronzio, Nucl. Phys. B136, 115 (1978).

[8] V. A. Rubakov and S. V. Troitsky, arXiv:hep-ph/0001213.

[9] C. Liu, Phys. Lett. B 591, 137 (2004).

[10] S. Weinberg, in General Relativity edited by S.W. Hawking and W. Israel (Cambridge University Press, Cambridge, England, 1979), pp. 790-831; M. Niedermaier and M. Reuter, Living Rev. Relativity 9, 5 (2006).

[11] M. Shaposhnikov and C. Wetterich, Phys. Lett. B 683, 196 (2010); M. E. Shaposhnikov, Teor. Mat. Fiz. 170, 280 (2012) [Theor. Math. Phys. 170, 229 (2012)].

[12] K. G. Chetyrkin and M. F. Zoller, J. High Energy Phys. 06 (2012) 033.

[13] A. A. Andrianov, D. Espriu, M. A. Kurkov, and F. Lizzi (to be published).

[14] See, e.g., A. Lenz, Adv. High Energy Phys. 2013, 910275 (2013).

[15] A. Connes and M. Marcolli, Noncommutative Geometry, Quantum Fields and Motives (American Mathematical Society, Providence, R.I., 2007); P. Aschieri, M. Dimitrijevich, P. Kulish, F. Lizzi, and J. Wess, Noncommutative Spacetimes: Symmetries in Noncommutative Geometry and Field Theory, Springer Lecture Notes in Physics Vol. 774 (Springer, Berlin, 2009).

[16] C. Rovelli, Quantum Gravity (Cambridge University Press, Cambridge, England, 2007). 